\title{
Ten-year experience with off-pump coronary artery bypass grafting: Lessons learned from early postoperative angiography
}

\author{
Ki-Bong Kim, MD, PhD, ${ }^{a}$ Jun Sung Kim, MD, ${ }^{c}$ Hyun-Jae Kang, MD, PhD, ${ }^{\mathrm{b}}$ Bon-Kwon Koo, MD, PhD, \\ Hyo-Soo Kim, MD, PhD, ${ }^{b}$ Byung-Hee Oh, MD, PhD, ${ }^{b}$ and Young-Bae Park, MD, PhD ${ }^{b}$
}

Objective: We performed early postoperative angiography to assess anastomosis accuracy and patency after offpump coronary artery bypass grafting.

\begin{abstract}
Methods: One thousand three hundred forty-five patients who underwent off-pump coronary artery bypass grafting between January 1998 and December 2007 were studied. Grafts for distal anastomoses were left internal thoracic artery $(n=1281)$, right internal thoracic artery $(n=679)$, right gastroepiploic artery $(n=836)$, radial artery $(\mathrm{n}=14)$, and saphenous vein $(\mathrm{n}=188)$. Groups underwent off-pump coronary artery bypass grafting without (group I, $\mathrm{n}=234$ ) or with (group II, $\mathrm{n}=1111$ ) intraoperative graft flowmetry. Early postoperative ( $\leq 7$ days) angiography was performed in 1278 cases $(95.0 \%)$ at $1.6 \pm 1.2$ postoperative days.
\end{abstract}

Results: Operative mortality was $1.6 \%$. Average number of distal anastomoses was $3.0 \pm 1.0$. Postoperative angiography showed early patencies of $98.9 \%$ for arterial grafts and $88.2 \%$ for venous grafts $(P<.001)$. In group II, intraoperative flowmetry-guided revision was performed in $2.2 \%$ of distal anastomoses. Patency of arterial grafts was significantly higher in group II than group I $(97.2 \%$ vs $99.1 \%, P<.001)$; however, patency of venous grafts was not significantly different $(86.0 \%$ vs $92.2 \%, P=.099)$. Early reoperation for graft revision according to angiographic findings was performed in 35 patients.

Conclusions: Early patency of venous grafts was significantly lower than that of arterial grafts. Intraoperative flowmetry and revision of abnormal grafts improved early graft patency, and reoperation according to early angiographic findings may further improve graft patency. (J Thorac Cardiovasc Surg 2010;139:256-62)

Because early graft patency can influence the outcome of coronary artery bypass grafting (CABG), there have been concerns about accuracy and patency of the grafts with the resurgent interest in off-pump CABG (OPCAB) ${ }^{1,2}$ Of several methods to assess graft patency, ultrasonographic flowmetry has been used with increasing frequency because of its noninvasiveness, simplicity, and reproducibility; however, it underestimates or overestimates the anastomotic accuracy relative to angiography. ${ }^{3-5}$ Although conventional angiography is the criterion standard technique, it has limitations as an intraoperative or early postoperative study. ${ }^{6,7}$ Despite the limitations of current methods of assessing graft patency, however, early identification of graft compromise can facilitate timely graft revision, thereby ensuring a patent graft at discharge. The aims of this study included (1) assessment of the early angiographic results of a 10-year OPCAB experi-

From the Departments of Thoracic and Cardiovascular Surgery ${ }^{\mathrm{a}}$ and Internal Medicine, ${ }^{\mathrm{b}}$ Seoul National University Hospital, and the Department of Thoracic and Cardiovascular Surgery, Boramae Medical Center, ${ }^{\mathrm{c}}$ Seoul, Republic of Korea. Disclosures: None.

Read at the Eighty-ninth Annual Meeting of The American Association for Thoracic Surgery, Boston, Mass, May 9-13, 2009.

Received for publication April 30, 2009; revisions received Aug 15, 2009; accepted for publication Aug 26, 2009.

Address for reprints: Ki-Bong Kim, MD, PhD, Department of Thoracic and Cardiovascular Surgery, Seoul National University Hospital, 28, Yeongeon-dong, Chongnogu, Seoul 110-744, Korea (E-mail: kimkb@snu.ac.kr).

$0022-5223 / \$ 36.00$

Copyright (c) 2010 by The American Association for Thoracic Surgery doi:10.1016/j.jtcvs.2009.08.040 ence, (2) evaluation of the efficacy of intraoperative graft flowmetry and early angiography, and (3) presentation of the results of early angiographically guided reinterventions.

\section{MATERIALS AND METHODS \\ Patient Characteristics}

Among the 1481 patients who underwent isolated CABG between January 1998 and December 2007, OPCAB was performed in 1345 cases $(90.8 \%)$. During the study period there were 38 conversions $(38 / 1383$, $2.7 \%$ ) to cardiopulmonary bypass during OPCAB, and those procedures were excluded from this study. Since the introduction of transit-time flow measurement (TTFM; Medi-Stim AS, Oslo, Norway) at our institution in 2000, we have derived criteria to predict abnormal grafts (occluded or competitive grafts) and revised the abnormal graft intraoperatively. ${ }^{8}$ The study patients were divided into group $\mathrm{I}(\mathrm{n}=234)$, patients who underwent OPCAB before the introduction of TTFM, and group II $(n=1111)$, patients who underwent OPCAB after the introduction of TTFM in 2000. There were no differences in sex, preoperative risk factors such as dyslipidemia and history of stroke, acute coronary syndrome, and left ventricular dysfunction (left ventricular ejection fraction $<35 \%$ measured by transthoracic echocardiography) between the groups. Other risk factors, however, such as hypertension $(P=.000)$ and diabetes $(P=.011)$, were more frequent in group II, and group II patients were also older than those in group I $(P=.001)$. The average number of distal anastomoses per patient was $3.0 \pm 1.0$, with no significant intergroup difference $(3.1 \pm 1.1$ in group I vs $3.0 \pm 1.0$ in group II, $P=.578)$. The grafts used for distal anastomoses were left internal thoracic artery (ITA, $n=1281)$, right ITA $(n=679)$, right gastroepiploic artery (RGEA, $n=836)$, radial artery $(n=14)$, and saphenous vein $(n=188)$. Left and right ITAs were used at similar frequencies, whereas RGEAs were used predominately in group II, and saphenous veins and radial arteries were used predominately in group I (Table 1). 


\section{Abbreviations and Acronyms \\ $\mathrm{CABG}=$ coronary artery bypass grafting \\ ITA $=$ internal thoracic artery \\ LAD $=$ left anterior descending coronary artery \\ $\mathrm{OPCAB}=$ off-pump coronary artery bypass grafting \\ RGEA = right gastroepiploic artery \\ TTFM $=$ transit-time flow measurement}

Early postoperative ( $\leq 7$ days) angiography for evaluation of the anastomotic sites and patency of the grafts was performed in 1278 patients $(95.0 \%)$ on postoperative $1.6 \pm 1.2$ days. Informed written consent, including the possible benefits and risks of postoperative angiography, was obtained from all patients. Patients who died, refused angiographic evaluation, or had postoperative development of acute renal failure were excluded from the angiographic follow-up. Patients with end-stage renal disease with long-term maintenance dialysis, however, were included in the angiographic follow-up.

Follow-up angiography included a selective graft angiogram with an additional native coronary angiogram taken when the graft angiogram revealed abnormal findings. All patients received continuous intravenous nitroglycerin until angiography was performed to avoid coronary spasm during the early postoperative period. If angiography demonstrated any abnormal finding, this was managed with medical follow-up, percutaneous intervention in the catheterization laboratory, or surgical intervention. Surgical intervention was indicated in cases with (1) abnormal findings including occlusion of the left anterior descending coronary artery (LAD) territory anastomosis, (2) occlusion of multiple distal anastomoses other than the LAD anastomosis, or (3) a graft trunk problem such as total or near total nonopacification of the proximal or middle graft trunk by graft angiography but well-visualized distal anastomoses and distal graft trunk by native coronary angiography.

The patients were continuously monitored with postoperative electrocardiography during stays in the intensive care unit and in the general ward until the chest tubes were removed. A standard 12-lead electrocardiogram was checked daily thereafter during the hospital stay. Occurrence of any short runs of atrial fibrillation during the hospital stay was considered to represent development of atrial fibrillation. Perioperative myocardial infarction was defined as positive results on at least 2 of 3 different tests: elevated peak serum creatine kinase isoenzyme level ( $>100 \mathrm{ng} / \mathrm{mL})$, appearance of new Q waves on the electrocardiogram, or newly developed regional wall motion abnormalities on the postoperative echocardiogram. Postoperative acute renal failure was defined as an increase of more than $50 \%$ in serum creatinine level from the preoperative value or a need for renal replacement therapy irrespective of serum creatinine level. Stroke was defined as a new and sudden onset of neurologic deficits lasting longer than 24 hours with no apparent nonvascular causes.

A computer-based patient database system was used for this retrospective study. The study protocol was reviewed by the institutional review board and approved as a minimal risk retrospective study (approval no. H-0812-053-266) that did not require individual consent according to the institutional guidelines for waiving consent.

\section{Surgical Techniques and Revascularization Strategies}

The basic surgical procedures and principles of OPCAB have been previously described. ${ }^{9}$ The operations were all performed by a single surgeon (K-B.K). The patients were heparinized with an initial $1.5 \mathrm{mg} / \mathrm{kg}$ dose of heparin and periodically received supplemental doses to maintain an activated clotting time longer than 300 seconds during OPCAB. During the study period, we changed revascularization strategies because of our early patency study after OPCAB.$^{10}$ Since the latter half of 1999 , we have tried to perform total arterial revascularization and to avoid the use of venous grafts if possible, a skeletonized technique for harvesting the arterial graft has been used, and protamine has not been given at the end of the procedure. Since 2000, we have revised the abnormal graft intraoperatively under a collective interpretation of TTFM variables. The criteria for predicting abnormal grafts ${ }^{8}$ were as follows: (1) systolic dominant or balanced pattern of the flow curve in the left coronary territories and systolic dominant pattern of the flow curve in the right coronary territories, (2) mean flow less than $15 \mathrm{~mL} / \mathrm{min}$, (3) pulsatility index greater than 3 in the left coronary territories and greater than 5 in the right coronary territories, and (4) insufficiency ratio greater than $2 \%$. After performing each anastomosis, we examined the anastomosis status with TTFM and revised it if there was any abnormal finding in TTFM. All the anastomoses were rechecked with TTFM after completion of revascularization just before pericardial closure.

All patients stopped taking aspirin on the day of surgery and resumed aspirin beginning at 1 postoperative day. Ticlopidine hydrochloride $(200 \mathrm{mg} /$ day) was used simultaneously with aspirin for 2 postoperative months. If the patient had a high blood level of low-density lipoprotein cholesterol $(>100 \mathrm{mg} / \mathrm{dL}$ ) postoperatively, drug therapy was started.

\section{Statistical Analysis}

Statistical analyses were performed with the SPSS software package (version 12.0; SPSS, Inc, Chicago, Ill). The significance of differences between groups was assessed with the unpaired 2-tailed $t$ test for continuous variables and with the $\chi^{2}$ test and Fisher's Exact Test for categoric variables. Patient demographic and clinical characteristics associated with revision reoperation were assessed by univariate analysis $\left(\chi^{2}, t\right.$ test $)$ and multivariable analysis (multiple logistic analysis). The univariate variables with $P$ values less than .3 were entered into the logistic regression to detect significant independent risk factors. All results were expressed as mean \pm SD for continuous data and proportion for binary data.

\section{RESULTS}

\section{Mortality and Morbidities}

Operative mortality (any death within 30 days, including deaths after hospital discharge) among all patients was 1.6\% (22/1345), and there was no difference between the groups ( $1.7 \%$ vs $1.6 \%, P$ not significant). Postoperative morbidities were atrial fibrillation $(23.9 \%)$, perioperative myocardial infarction $(5.7 \%)$, acute renal failure (2.8\%), bleeding-related reoperation $(3.9 \%)$, reoperation for graft revision $(2.6 \%)$, stroke $(1.6 \%)$, and mediastinitis $(1.2 \%$; Table 2$)$. There were statistically significant differences between the groups in atrial fibrillation $(P<.001)$, acute renal failure $(P=.049)$, and graft revision-related reoperation $(P<.001)$. The higher incidences of atrial fibrillation and acute renal failure in group II may be related to differences between the groups in risk factors such as hypertension, diabetes, and elderly age.

Among the postoperative morbidities, 5 of 21 cases of stroke $(5 / 1278,0.4 \%)$ and 11 of 38 cases of postoperative acute renal failure $(11 / 1278,0.9 \%)$ were suspected to be related to postoperative angiography. All 5 cases of catheterization-related stroke were of minor degree, and only 1 of the 11 patients with contrast medium-induced nephropathy required temporary renal replacement therapy. 
TABLE 1. Preoperative characteristics of the patients and grafts used

\begin{tabular}{|c|c|c|c|c|}
\hline & Total $(n=1345)$ & Group I $(n=234)$ & Group II $(n=1111)$ & $P$ value \\
\hline Age $(y$, mean $\pm S D)$ & $63 \pm 10$ & $61 \pm 9$ & $64 \pm 10$ & .001 \\
\hline Female (no.) & $397(29.5 \%)$ & $73(31.1 \%)$ & $324(29.2 \%)$ & .535 \\
\hline Smoking (no.) & $641(47.7 \%)$ & $110(47.0 \%)$ & $531(47.8 \%)$ & .817 \\
\hline Hypertension (no.) & $902(67.0 \%)$ & $133(56.8 \%)$ & $769(69.2 \%)$ & $<.001$ \\
\hline Diabetes (no.) & $607(45.1 \%)$ & $88(37.6 \%)$ & $519(46.7 \%)$ & .011 \\
\hline Dyslipidemia (no.) & $346(25.7 \%)$ & $52(22.2 \%)$ & $294(26.5 \%)$ & .177 \\
\hline History of stroke (no.) & $163(12.1 \%)$ & $25(10.7 \%)$ & $138(12.4 \%)$ & .459 \\
\hline Previous percutaneous coronary intervention (no.) & $182(13.6 \%)$ & $35(15.0 \%)$ & $147(13.3 \%)$ & .489 \\
\hline Chronic renal failure (no.) & $102(7.6 \%)$ & $15(6.4 \%)$ & $87(7.8 \%)$ & .456 \\
\hline Chronic obstructive pulmonary disease (no.) & $10(0.7 \%)$ & $3(1.3 \%)$ & $7(0.6 \%)$ & .157 \\
\hline Left ventricular ejection fraction $<35 \%$ (no.) & $123(9.1 \%)$ & $25(10.7 \%)$ & $98(8.8 \%)$ & .371 \\
\hline Left main or 3-vessel disease (no.) & $568(42.2 \%)$ & $104(44.4 \%)$ & $464(41.8 \%)$ & .108 \\
\hline Acute coronary syndrome (no.) & $962(71.5 \%)$ & $163(69.7 \%)$ & $799(71.9 \%)$ & .486 \\
\hline Left internal thoracic artery (no.) & $1281(95.2 \%)$ & $224(95.7 \%)$ & $1057(95.1 \%)$ & .701 \\
\hline Right internal thoracic artery (no.) & $679(50.5 \%)$ & $113(48.3 \%)$ & $566(50.9 \%)$ & .460 \\
\hline Right gastroepiploic artery (no.) & $836(62.2 \%)$ & $47(20.1 \%)$ & $789(71.0 \%)$ & $<.001$ \\
\hline Radial artery (no.) & $14(1.0 \%)$ & $8(4.9 \%)$ & $6(0.5 \%)$ & $<.001$ \\
\hline Saphenous vein (no.) & $188(14.0 \%)$ & $108(46.2 \%)$ & $80(7.2 \%)$ & $<.001$ \\
\hline
\end{tabular}

\section{Early Angiographic Results of 10-Year OPCAB Experience (Table 3)}

Of the 1345 patients, 1278 patients $(95.0 \%)$ underwent early postoperative angiography on postoperative $1.6 \pm 1.2$ days. A total of 3857 distal anastomoses were evaluated. Arterial graft patency was $98.9 \%$, which was higher than saphenous vein graft patency $(88.2 \%$, $P<.001)$. In group I, arterial graft patency was $97.2 \%$ and saphenous vein graft patency was $86.0 \%$. Among group II patients who underwent TTFM examination, arterial graft and saphenous vein graft patency rates improved to $99.1 \%$ and $92.2 \%$, respectively. Between the groups, arterial patency rate was improved with statistical significance after intraoperative flowmetry (arterial patency $P<.001$, venous patency $P=.099$ ). For revascularized territories, patency rates of the LAD and left circumflex artery territories were both $98.2 \%$ and that of the right coronary artery territory was $97.1 \%$ in the combined group of total patients, but these differences were not significant.

\section{Intraoperative Graft Revision Guided by TTFM}

Since the introduction of TTFM at our institution, there have been 118 revisions in 110 patients (110 of 1111 patients, $9.9 \%$ ). The causes of revision were as follows: (1) distal anastomotic failures by thrombosis, stenosis, kinking, coronary or graft dissection, or unknown causes (75 failures in 73 patients, 75/3343 anastomoses [2.2\%], 73/1111 patients [6.6\%]); (2) graft trunk problems without distal anastomotic failures, such as dissection $(\mathrm{n}=3)$, anastomotic thrombosis of composite graft $(\mathrm{n}=1)$, and torsion $(\mathrm{n}=1)$; and (3) competitive flow patterns $(\mathrm{n}=38)$. Two thirds of revisions for competitive flow patterns $(27 / 38,71.1 \%)$ occurred in revascularization of the right coronary artery territory with an in situ RGEA graft. If there was suspected flow competition, the in situ RGEA was divided at its proximal section and anastomosed to the side of the left ITA in a Y-composite fashion or to the side of the ascending aorta. Distal anastomotic revision rates in the LAD, left circumflex artery, and right coronary artery territories were similar

TABLE 2. Postoperative morbidity and mortality

\begin{tabular}{|c|c|c|c|c|c|c|c|}
\hline & \multicolumn{2}{|c|}{ Total $(n=1345)$} & \multicolumn{2}{|c|}{ Group I $(n=234)$} & \multicolumn{2}{|c|}{ Group II $(n=1111)$} & \multirow[b]{2}{*}{$P$ value } \\
\hline & No. & $\%$ & No. & $\%$ & No. & $\%$ & \\
\hline Mortality $(<30 \mathrm{~d})$ & 22 & $1.6 \%$ & 4 & $1.7 \%$ & 18 & $1.6 \%$ & $>.999$ \\
\hline \multicolumn{8}{|l|}{ Morbidity } \\
\hline Atrial fibrillation & 321 & $23.9 \%$ & 25 & $10.7 \%$ & 296 & $26.6 \%$ & $<.001$ \\
\hline Perioperative myocardial infarction & 76 & $5.7 \%$ & 11 & $4.7 \%$ & 65 & $5.9 \%$ & .462 \\
\hline Stroke & 21 & $1.6 \%$ & 4 & $1.7 \%$ & 17 & $1.5 \%$ & .776 \\
\hline Acute renal failure & 38 & $2.8 \%$ & 2 & $0.9 \%$ & 36 & $3.2 \%$ & .05 \\
\hline Mediastinitis & 16 & $1.2 \%$ & 2 & $0.9 \%$ & 14 & $1.3 \%$ & $>.999$ \\
\hline Bleeding reoperation & 52 & $3.9 \%$ & 6 & $2.6 \%$ & 46 & $4.2 \%$ & .243 \\
\hline Revision reoperation & 35 & $2.6 \%$ & 15 & $6.4 \%$ & 20 & $1.8 \%$ & $<.001$ \\
\hline
\end{tabular}


TABLE 3. Postoperative angiographic patency rates by graft and by target coronary artery territory

\begin{tabular}{|c|c|c|c|c|c|c|c|}
\hline & \multicolumn{2}{|c|}{ Total $(\mathbf{n}=1278)$} & \multicolumn{2}{|c|}{ Group I $(\mathbf{n}=221)$} & \multicolumn{2}{|c|}{ Group II $(n=1047)$} & \multirow[b]{2}{*}{$P$ value } \\
\hline & No. & $\%$ & No. & $\%$ & No. & $\%$ & \\
\hline \multicolumn{8}{|l|}{ By graft } \\
\hline Arterial graft & $3495 / 3535$ & $98.9 \%$ & $452 / 465$ & $97.2 \%$ & $3043 / 3070$ & $99.1 \%$ & $<.001$ \\
\hline Internal thoracic & $2218 / 2239$ & $99.1 \%$ & $395 / 407$ & $97.1 \%$ & $1823 / 1832$ & $99.5 \%$ & $<.001$ \\
\hline Right gastroepiploic & $1259 / 1278$ & $98.5 \%$ & $45 / 46$ & $97.8 \%$ & $1214 / 1232$ & $98.5 \%$ & .504 \\
\hline Radial & $18 / 18$ & $100 \%$ & $12 / 12$ & $100 \%$ & $6 / 6$ & $100 \%$ & \\
\hline Venous graft & $284 / 322$ & $88.2 \%$ & $178 / 207$ & $86.0 \%$ & $106 / 115$ & $92.2 \%$ & .099 \\
\hline Total & $3779 / 3857$ & $98.0 \%$ & $630 / 672$ & $93.8 \%$ & $3149 / 3185$ & $98.9 \%$ & $<.001$ \\
\hline \multicolumn{8}{|c|}{ By target coronary artery territory } \\
\hline Left anterior descending & $1827 / 1860$ & $98.2 \%$ & $319 / 338$ & $94.4 \%$ & $1508 / 1522$ & $99.1 \%$ & $<.001$ \\
\hline Left circumflex & $1170 / 1192$ & $98.2 \%$ & $180 / 194$ & $92.8 \%$ & $990 / 998$ & $99.2 \%$ & $<.001$ \\
\hline Right coronary & $782 / 805$ & $97.1 \%$ & $131 / 140$ & $93.6 \%$ & $651 / 665$ & $97.9 \%$ & .010 \\
\hline
\end{tabular}

(2.5\% [40/1602] vs $2.2 \%$ [23/1045] vs $1.7 \%$ [12/696], $P$ not significant). Early angiographic results demonstrated that 71 of 75 distal anastomosis revisions (94.7\%) and all 5 graft trunk revisions were patent. Four of 8 distal anastomoses that showed persistently abnormal TTFM findings even after revision proved to be patent on early postoperative angiography.

\section{Early Angiographically Guided Reinterventions}

Thirty-eight patients needed reinterventions according to the early postoperative angiographic findings; 35 of these patients underwent reoperations, and the other 3 patients underwent percutaneous interventions. Of the 35 patients who underwent reoperations, group II patients showed a lower incidence of reoperation than group I patients $(6.4 \%$ [15/234] vs $1.8 \%$ [20/1111], $P<.001)$. The presence of early angiographic abnormalities that necessitated reoperation did not necessarily presage coronary compromise. None of the 35 patients had anginal symptoms or hemodynamic instability, other than perioperative myocardial infarction in 7 patients. Angiographic reasons for reoperation were as follows: abnormal findings, including occlusion of the LAD territory anastomosis $(\mathrm{n}=16)$; multiple anastomosis failures $(\mathrm{n}=2)$; and graft trunk problems $(\mathrm{n}=17)$. In group I, most reoperations $(14 / 15)$ were related to distal anastomotic occlusions ( 7 arterial graft occlusions, 5 venous graft occlusions, and 2 occlusions of both graft types). Another cause of reoperation was arterial graft trunk kinking. In contrast, 16 of 20 reoperations in group II were related to graft trunk problems. The causes of graft trunk problems in group II were delayed dissection of the arterial graft trunk $(n=6)$, graft trunk kinking $(n=6)$, localized thrombus around damaged intimal area $(n=2)$, composite graft stenosis $(n=1)$, and graft compression by localized hematoma $(n=1)$. Angiography-induced graft spasm was suspected in 1 case because we failed to find the cause of a severely stenotic long segment of ITA graft, which proved to be patent on reoperation. The other cause of 3 reoperations in group II was distal anastomotic occlusion of arterial grafts. Revision reoperations were performed on postoperative $1.9 \pm 1.5$ days, and 2 thirds of reoperations $(24 / 35,68.6 \%)$ were performed on the day of angiography. Among reoperations, 7 patients needed cardiopulmonary bypass and 16 patients underwent OPCAB for distal anastomotic revision; 12 patients with graft trunk problems underwent a correction without cardiac manipulation or distal anastomotic revision. Early angiographic findings demonstrated an overall patency rate of $44.1 \%(52 / 118)$ among 35 patients who underwent revision reoperation. In 27 patients, graft patency was re-evaluated by angiography after reoperation and before discharge; this showed a $96.4 \%$ patency rate $(53 / 55)$ of revised grafts.

Three patients underwent percutaneous interventions instead of reoperation: 2 patients with significant stenosis of LAD anastomosis and 1 patient with occlusion of the obtuse marginal branch anastomosis. We attempted to identify the risk factors in reinterventions performed in 38 patients. Univariate analysis demonstrated that, without using an intraoperative TTFM, multiple distal anastomoses and saphenous vein graft use were risk factors. Multivariable logistic regression analysis showed lack of intraoperative TTFM as an independent risk factor (odds ratio 4.237, $P<.001$; Table 4).

\section{DISCUSSION}

This study revealed 3 main findings. First, the early postoperative patency rate of venous grafts after OPCAB was significantly lower than that of arterial grafts. Second, intraoperative graft TTFM and revision of abnormal grafts improved early graft patency rates. Third, $1.8 \%$ of patients required reoperation despite of intraoperative TTFM-guided revision, and reoperation according to early angiographic findings probably further improved graft patency.

There have been concerns about accuracy and patency of grafts made during OPCAB because of the technical difficulties of anastomosis caused by the cardiac motion, lack of a bloodless operative field, and poor exposure of target vessels. Although technical improvements and development of operative devices such as the mechanical stabilizer have resulted in graft patency results after OPCAB similar to those 
TABLE 4. Risk factor analysis for early angiographically guided reinterventions

\begin{tabular}{|c|c|c|c|c|}
\hline & \multirow[b]{2}{*}{ Univariate analysis $P$ value } & \multicolumn{3}{|c|}{ Multivarible analysis } \\
\hline & & $P$ value & Odds ratio & $95 \% \mathrm{CI}$ \\
\hline Without transit-time flow measurement & $<.001$ & $<.001$ & 4.237 & $2.118-8.476$ \\
\hline Smoking & .640 & & & \\
\hline Diabetes mellitus & .353 & & & \\
\hline Hypertension & .337 & & & \\
\hline Dyslipidemia & .050 & .088 & 0.398 & $0.138-1.147$ \\
\hline Female & .598 & & & \\
\hline Age & .576 & & & \\
\hline Chronic renal failure & .508 & & & \\
\hline Previous percutaneous coronary intervention & .807 & & & \\
\hline Acute coronary syndrome & 698 & & & \\
\hline Left main or 3-vessel disease & .354 & & & \\
\hline Left ventricular ejection fraction $<35 \%$ & .753 & & & \\
\hline Emergency or urgent operation & .706 & & & \\
\hline Distal anastomosis $\geq 4$ & .048 & .057 & 1.959 & $0.980-3.915$ \\
\hline Venous graft & .001 & .187 & 1.769 & $0.758-4.128$ \\
\hline Right gastroepiploic artery graft & .080 & .828 & 0.904 & $0.364-2.246$ \\
\hline
\end{tabular}

with conventional $\mathrm{CABG},{ }^{1,12}$ other studies have demonstrated lower graft patency rates after OPCAB than after conventional CABG. ${ }^{10,13,14}$ Those differences were mainly attributable to the lower patency rates of saphenous vein grafts. A significantly lowered patency for saphenous vein grafts relative to ITA grafts has been suggested to result from the type of graft, presence of dyslipidemia, exposure and quality of stabilization, and increased procoagulatory activity in OPCAB patients. ${ }^{10,15,16}$ A previous study that used early postoperative angiography ${ }^{17}$ demonstrated an overall graft patency rate among patients who received venous grafts significantly lower than that among patients who received total arterial grafts. Multivariable logistic regression analysis failed to demonstrate early experience as an independent predictor, and venous graft use itself was an independent predictor of graft failure during the first postoperative year after OPCAB. In this study, saphenous vein grafts had significantly lower patency rates than did arterial grafts on early postoperative angiography $(P<.001)$. After the introduction of intraoperative TTFM, patency rates of arterial grafts improved with statistical significance $(P<.001)$; however, patency rates of saphenous vein grafts did not improve significantly $(P=.099)$. This suggests that the lowered patency rates of saphenous vein grafts may result from the type of graft, although the relatively small number of venous grafts involved in this study may also have affected the results.

In addition to the type of graft, immediate intraoperative graft failure, most commonly resulting from technical errors, is another major factor that determines the early graft patency after revascularization. ${ }^{18,19}$ The natural incidence of intraoperative graft failure has been poorly documented, however, because most surgeons perform CABG without any immediate confirmation of a technical result. Several methods, including manual palpation of the graft, direct probing of the anastomosis, graft patency testing with syringe, electromagnetic flowmetry, ultrasonographically based flowmetry (such as Doppler and TTFM), and intraoperative angiography (such as conventional angiography and fluorescence imaging), have been used to assess graft patency intraoperatively. Although conventional angiography is the criterion standard technique for assessing graft patency, it is rarely available in the operating room because of its requirements for additional equipment and personnel. Among those methods for assessing intraoperative graft patency, TTFM has been used with increasing frequency because it is considered to be a convenient and reliable way to document graft patency. ${ }^{8,18}$ D'Ancona and colleagues ${ }^{18}$ reported that overall $3.2 \%$ of 1145 grafts in $7.6 \%$ of 406 patients undergoing OPCAB were revised on the basis of TTFM findings. Although some studies ${ }^{3-5}$ have indicated that TTFM is likely to underestimate or overestimate the need for graft revision in comparison with angiography, we found it useful to predict graft abnormality with a high sensitivity and to improve graft patency by revising the anastomosis under a collective interpretation of TTFM variables. $^{8}$ In this study, 75 of 3343 distal anastomoses $(2.2 \%)$ and 5 graft trunk problems required revisions, which may prove of help in avoiding the adverse outcomes caused by poor graft patency. Four of 8 distal anastomoses that showed persistently abnormal TTFM findings even after revision proved to be patent on early postoperative angiography, suggesting graft spasm or local edema at the anastomosis site as a cause of the false-positive findings $(4 / 75,5.3 \%)$. We believe that intraoperative identification of graft compromise facilitated timely graft revision by 
surgical intervention, thereby ensuring a patent graft and excellent flow before chest closure.

Causes of immediate postoperative graft failure other than intraoperative technical errors that contribute to the early graft patency have rarely been described in previous studies. With the mechanical manipulation associated with surgery and the current harvesting techniques of skeletonized arterial grafts, along with a possible hypercoagulable state in the first 24 hours after OPCAB,${ }^{15,20}$ there is a potential for vascular trauma that could induce vasoconstriction, edema, intramural hematoma or dissection, thrombosis, or kinking of the graft trunk after pericardial closure. These factors may cause additional immediate postoperative graft failure. In this study, there were 35 patients (35/ $1278,2.7 \%$ ) who needed reoperation on the basis of early postoperative angiographic findings. Although the incidence of reoperation decreased after the introduction of TTFM, there were still $1.8 \%$ of patients who required reoperation on the basis of early angiography despite intraoperative TTFM-guided revision. Univariate analysis showed that the annual incidence of reoperation after the introduction of TTFM $(1.4 \%$ to $2.6 \%)$ was not influenced by time factor (odds ratio $0.95, P=.653$ ). We believe that early postoperative identification of graft compromise also facilitates timely graft revision by surgical intervention, thereby ensuring a patent graft at the time of discharge, although it is not known whether early graft patency translates into long-term graft patency. Another advantage of early postoperative angiography is direct feedback for quality control and technical improvement when adopting a new, technically demanding surgical procedure such as OPCAB. An additional advantage of early reoperation on the basis of early angiography was the reusability of failed grafts in most of our cases when graft compromise occurred in arterial grafts.

Although angiography is considered the criterion standard for morphologic assessment of native coronary arteries and bypass grafts, it has limitations as an early postoperative study. First, there are difficulties in the interpretation of anastomotic stenosis visualized during the early angiogram. Several studies ${ }^{6,7,21}$ demonstrated that a majority of anastomotic stenoses or spastic arterial grafts seen in early angiograms became patent on later follow-up, which may result from local tissue edema or vasospasm. This study did not include anastomotic stenosis as an indication for reoperation. Second, postoperative morbidities such as stroke and acute renal failure may develop as a result of catheterization or use of contrast medium. In this study, $0.4 \%$ of strokes and $0.9 \%$ of postoperative acute renal failures were suspected to be related to postoperative angiography. Although all the patients with angiography-related stroke and nephropathy recovered without major sequelae, we have modified our protocol of postoperative angiography as follows: catheterization through a radial artery for pressure monitoring, heparinization to maintain an activated clotting time of more than 180 seconds during the angiogram, and hydration and use of $\mathrm{N}$-acetylcysteine to reduce the incidence of contrast medium-induced nephropathy.

Some limitations to this study must be recognized. First, this was a retrospective observational study, although all experiences were at a single institution and were collected with a computer-based database system. Second, we changed the revascularization strategies during the study period. Most patients who received venous grafts underwent OPCAB in the early study period, and most patients who received total arterial grafts underwent OPCAB in the latter part of the study period. In addition, the annual incidence of reoperation during the whole study period was influenced by time factor (odds ratio $0.82, P=.014$ ), although the annual incidence of reoperation after the introduction of TTFM was not influenced by time factor (odds ratio 0.95 , $P=.653$ ). Both things would affect the difference between the groups.

\section{References}

1. Khan NE, De Souza A, Mister R, Flather M, Clague J, Davies S, et al. A randomized comparison of off-pump and on-pump multivessel coronary-artery bypass surgery. N Engl J Med. 2004;350:21-8.

2. Parolari A, Alamanni F, Polvani G, Agrifoglio M, Chen YB, Kassem S, et al. Meta-analysis of randomized trials comparing off-pump with on-pump coronary artery bypass graft patency. Ann Thorac Surg. 2005;80:2121-5.

3. Hirotani T, Kameda T, Shirota S, Nakao Y. An evaluation of the intraoperative transit time measurements of coronary bypass flow. Eur J Cardiothorac Surg. 2001;19:848-52.

4. Balacumaraswami L, Abu-Omar Y, Choudhary B, Pigott D, Taggart DP. A comparison of transit-time flowmetry and intraoperative fluorescence imaging for assessing coronary artery bypass graft patency. J Thorac Cardiovasc Surg. 2005; 130:315-20.

5. Tokuda Y, Song MH, Ueda Y, Usui A, Akita T. Predicting early coronary artery bypass graft failure by intraoperative transit time flow measurement. Ann Thorac Surg. 2007;84:1928-33.

6. Wiklund L, Johansson M, Brandrup-Wognsen G, Bugge M, Radberg G, Berglin E. Difficulties in the interpretation of coronary angiogram early after coronary artery bypass surgery on the beating heart. Eur J Cardiothorac Surg. 2000; 17:46-51.

7. Shin H, Yozu R, Mitsumaru A, Iino Y, Hashizume K, Matayoshi T, Kawada S Intraoperative assessment of coronary artery bypass graft: transit-time flowmetry versus angiography. Ann Thorac Surg. 2001;72:1562-5.

8. Kim K-B, Kang CH, Lim C. Prediction of graft flow impairment by intraoperative transit time flow measurement in off-pump coronary artery bypass using arterial grafts. Ann Thorac Surg. 2005;80:594-9.

9. Kim K-B, Cho KR, Chang WI, Lim C, Ham BM, Kim YL. Bilateral skeletonized internal thoracic artery graftings in off-pump coronary artery bypass: early result of Y versus in situ grafts. Ann Thorac Surg. 2002;74:S1371-6.

10. Kim K-B, Lim C, Lee C, Chae IH, Oh BH, Lee MM, Park YB. Off-pump coronary artery bypass may decrease the patency of saphenous vein grafts. Ann Thorac Surg. 2001;72:S1033-7.

11. Lingaas PS, Hol PK, Lundbald R, Rein KA, Mathisen L, Smith HJ, et al. Clinical and radiologic outcome of off-pump coronary surgery at 12 months follow-up: a prospective randomized trial. Ann Thorac Surg. 2006;81:2089-96.

12. Al-Ruzzeh S, George S, Bustami M, Wray J, Ilsley C, Athanasiou T, et al. Effect of off-pump coronary artery bypass surgery on clinical, angiographic, neurocognitive, and quality of life outcomes: randomized controlled trial. BMJ. 2006;332: 1365

13. Lim E, Drain A, Davies W, Edmonds L, Rosengard BR. A systematic review of randomized controlled trials comparing revascularization rate and graft patency of off-pump and conventional coronary surgery. J Thorac Cardiovasc Surg. 2006; 132:1409-13. 
14. Takagi H, Tanabashi T, Kawai N, Kato T, Umemoto T. Off-pump coronary artery bypass sacrifices graft patency: meta-analysis of randomized trials. J Thorac Cardiovasc Surg. 2007;133:e2-3.

15. Mariani MA, Gu YJ, Boonstra PW, Grandjean JG, van Oeveren W, Ebels T. Procoagulant activity after off-pump coronary operation: Is the current anticoagulation adequate? Ann Thorac Surg. 1999;67:1370-5

16. Ömeroğlu SN, Kırali K, Güler M, Toker ME, İpek G, Işık Ö, et al. Midterm angiographic assessment of coronary artery bypass grafting without cardiopulmonary bypass. Ann Thorac Surg. 2000;70:844-9.

17. Cho KR, Jeong DS, Kim K-B. Influence of vein graft use on postoperative 1-year results after off-pump coronary artery bypass surgery. Eur J Cardiothorac Surg. 2007;32:718-23.

18. D'Ancona G, Karamanoukian HL, Ricci M, Schmid S, Bergsland J, Salerno TA. Graft revision after transit time flow measurement in off-pump coronary artery bypass grafting. Eur J Cardiothorac Surg. 2000;17:287-93.

19. Hol PK, Lingaas PS, Lundblad R, Rein KA, Vatne K, Smith HJ, et al. Intraoperative angiography leads to graft revision in coronary artery bypass surgery. Ann Thorac Surg. 2004;78:502-5.

20. Mack MJ, Magovern JA, Acuff TA, Landreneau RJ, Tennison DM, Tinnerman EJ, et al. Results of graft patency by immediate angiography in minimally invasive coronary artery surgery. Ann Thorac Surg. 1999;68:383-9.

21. Kim K-B, Cho KR, Jeong DS. Midterm angiographic follow-up after off-pump coronary artery bypass: serial comparison using early, 1-year, and 5-year postoperative angiograms. J Thorac Cardiovasc Surg. 2008;135:300-7.

\section{Discussion}

Dr Joseph F. Sabik (Cleveland, $\mathrm{OH}$ ). Dr Kim and colleagues report on their 10-year experience with early ITA and saphenous vein graft patency after OPCAB. Their observations provide insight into the early results of OPCAB and the roles that intraoperative and postoperative graft assessment may have in improving the results of surgical revascularization.

My first question, Dr Kim, has to do with your low early saphenous vein graft patency. In sharp contrast to the early saphenous vein graft patency observed by others such as Chesebro, who reported a 98\% saphenous vein graft patency at 1 week after surgery, you report only an $88.2 \%$ patency at 1.6 days after surgery. In other words, a little over 1 in 10 of your saphenous vein grafts have occluded within 2 days of surgery despite intraoperative assessment. This is both sobering and alarming, considering that other revascularization options exist such as arterial grafts—or, do I even mention, stents.

My first question is this: have your postoperative angiograms provided insight into the mechanism of failure of 1 in 10 of your saphenous vein grafts, or do you still believe, as you wrote in 2001, that saphenous vein grafts occlude after OPCAB because of a possible hypercoagulable state?

Dr Kim. Thank you, Dr Sabik, for your comments and excellent questions. During the study period, we changed revascularization strategies in response to our early patency study after OPCAB. Since the latter half of 1999, we have tried to perform total arterial revascularization and have avoided the use of venous grafts if possible. Venous grafts were used for patients who had limitations in using the ITA because of significant stenosis of the proximal subclavian artery, who underwent emergency operation, or who had a shortage of arterial grafts during total arterial revascularization. After the introduction of intraoperative TTFM, the patency rates of arterial grafts improved with statistical significance. The patency rates of venous grafts, however, did not improve significantly. This suggests that the lowered patency rates of venous grafts may result from the type of graft, although the relatively small number of venous grafts involved in this study may also have affected the results.

Impressed, however, by the elegant study of Dr Souza published in the Journal in 2006, which demonstrated an excellent patency rate of saphenous vein graft harvested with the no-touch technique, we are currently expanding the use of the saphenous vein graft. I hope I can present the revival of the saphenous vein graft in the near future.

Dr Sabik. But any thoughts on why the grafts are closing? Did your angiography demonstrate kinks in the vein grafts, dissections? Any idea why so many closed so early?

Dr Kim. Most of the saphenous vein graft failures occurred in the earlier part of our experience, without the use of TTFM, and most reoperations were related to distal anastomotic occlusions.

Dr Sabik. I thought it didn't change, though, from group I to group II statistically in venous graft failure?

Dr Kim. As I showed in my slide, there was an insignificant difference in venous graft patency between the groups. In the latter half of our experience, however, most of the graft failures were related to graft trunk problem, not distal anastomotic failure.

Dr Sabik. You currently assess your grafts intraoperatively with TTFM and postoperatively with angiography. Your intraoperative assessments led to graft revision in $9.9 \%$ of your patients, and your postoperative angiography led to coronary reoperation or reintervention in about $3 \%$. Because angiography is both more sensitive and specific than TTFM, why not do your angiography intraoperatively instead of waiting, so that you could correct whatever problem at the initial operation?

Dr Kim. Although conventional angiography is the criterion standard technique, it has limitations as an intraoperative study. It is rarely available in the operating room because of its requirement for additional facilities and personnel. Intraoperative angiography was not available at our institute during the study period.

In addition, there is an important lesson learned from early postoperative angiography. Causes of immediate postoperative graft failure other than intraoperative technical errors have rarely been discussed in previous studies. With the mechanical manipulation associated with surgery and the current harvesting technique of skeletonization, along with a possible hypercoagulable state in the first 24 hours after OPCAB, there is a potential for vascular trauma that could induce vasoconstriction, edema, intramural hematoma or dissection, thrombosis, or kinking of the graft after pericardial closure. These factors may cause additional immediate postoperative graft failure.

Although the incidence of reoperation decreased after introduction of TTFM, there were still $1.8 \%$ of patients who required reoperation according to early angiography. We do believe that early postoperative identification of graft compromise also facilitates timely graft revision by surgical intervention, thereby ensuring a patent graft at the time of the patient's discharge. 